\title{
Research on Mechanism of Distribution Line Failure Caused By Violent Typhoon
}

\author{
De-Hua CAI ${ }^{1, a}$, Jian-Wei WU ${ }^{2, b}$, Jian-Feng WEN ${ }^{1, c}$ and Xue-Jiao HAN ${ }^{3, d_{*}}$ \\ ${ }^{1}$ Jiangmen Power Supply Company of Guangdong Power Grid Co., Ltd Guangdong 529000, China \\ ${ }^{2}$ Guangdong Power Grid Co., Ltd Guangdong 510000, China \\ ${ }^{3}$ Tsinghua Universities, Beijing 100084, China \\ atsdd7219@21cn.com, ${ }^{\mathrm{b}} 89582823 @ q q . c o m,{ }^{\mathrm{c}} 79581819 @ q q . c o m,{ }^{d}$ xuejiao-han@tsinghua.edu.cn \\ *Corresponding author
}

Keywords: Electrical failure, Lightning stroke, Wind age yaw flashover, Wet flashover, Mechanical failure

\begin{abstract}
Distribution line failure caused by violent typhoon is studied in the paper. Violent typhoon is always accompanied by wind, rain as well as even thunder and lightning. Distribution line failure is sorted in the paper. Distribution line failures are divided into two major categories of electrical failure and mechanical failure according to mechanism. Lightning-caused lightning stroke, wind-caused wind age yaw flashover and rain-caused insulation wet flashover are mainly studied aiming at electrical failure. Tower damage and foundation damage are mainly analyzed aiming at mechanical failure. The research results of the paper can provide theoretical basis for analyzing distribution line failure in violent typhoon disaster regions.
\end{abstract}

\section{Introduction}

Disastrous typhoon weather produces huge operation risk and economic loss to electricity system. For example, 'Hagupit' typhoon landed in Guangdong Province in 2008, the wind force near the center of the landfall was grade 15 , there were many trips of $110 \mathrm{kV}$ or above lines, and most medium-voltage distribution lines were shut down. A total of $525.8 \mathrm{~km}$ of $10 \mathrm{kV}$ distribution lines were damaged in Yangjiang Distribution Network, and 4997 units suffered from pole falling and pole breaking. 2230 units suffered from pole falling and pole breaking in Maoming 10kV distribution line. 2615 units suffered from pole falling and pole breaking in Zhanjiang Distribution Network.Violent, typhoon 'Wipha' landed in Zhejiang Province in 2007 with the maximum wind speed exceeding 45m/s. 410 units in landing destination- Cangnan County along the $10 \mathrm{kV}$ distribution line suffered from pole falling and pole breaking. Medium-low pressure distribution line was damaged for $146 \mathrm{~km}$, and the direct economic loss exceeded 12.5 million Yuan. Violent typhoon Saomai landed from Wenzhou in 2006 with the maximum wind speed reaching $68 \mathrm{~m} / \mathrm{s}$, two $500 \mathrm{kV}$ transmission lines and three $220 \mathrm{kV}$ transmission lines were tripped, $51010 \mathrm{kV}$ distribution lines lost power due to destructive failure, more than 10,000 units suffered from pole falling and pole breaking, and economic losses were nearly 200 million Yuan. Hurricane Sandy hit the United States in 2012, 8 million households lost power, the power grid was restored for months, and the economy lost was $\$ 50$ billion. As climate warms up and disastrous weather here gets more frequent all over the world, wind disaster has become a major factor harming the safety of the power grid.

In recent years, violent typhoon landing has occurred frequently in China coastal areas. Although the meteorological department has issued an early warning message before the typhoon landing, the power department is usually lack of assessment to its harmfulness, and the security and defense measures are not enough. The modern society is becoming more and more dependent on the power system. The large-area failure of the power system brings huge economic loss to the society. The large-scale paralysis of the distribution system caused by several violent typhoon weathers indicates that the power system has severe defects to extreme weather conditions. Therefore, it is more and 
more urgent to study the distribution line failure coupling relationship caused by multiple meteorological processes.

\section{Electrical Failure}

\section{Lightning Stroke}

Lightning stroke is one of main causes of distribution line failure especially in areas where thunder and lightning activity is intense. There are two main ways of flashovers caused by overvoltage due to thunder and lightning according to the principle of thunder and lightning action: thunder and lightning directly strike distribution line to generate overvoltage and lightning induced overvoltage of lightning stroke overhead line near the ground. Even if the insulation level, conductor span and grounding of the distribution network transmission lines all reach the optimal conditions, and direct lightning stroke is more likely to cause transmission line insulation flashover without any lighting overvoltage protection. However, urban areas rarely suffer from direct lightning accident generally as a result of the shielding effect of buildings and trees, etc. Urban distribution line failure is mainly caused by the following reason: lightning induced overvoltage. Both direct lightning or thunder and lightning induced overvoltage pose a serious threat to the safe and stable operation of the equipment for transmission and transformation of power distribution network.

Statistics show that the probability of thunder and lightning flow amplitude greater than 200kA is 0.0053 and 2.85e-5 respectively for general areas. The probability of occurrence is already very low. Therefore, the thunder and lightning flow with amplitude greater than 200kA is ignored in the paper. The lightning ranges under different thunder and lightning flows are different according to electric geometric model. The same lighting range is selected for different thunder and lightning flows during previous calculation of lightning stroke trip rate. Errors are produced for calculation results. Therefore, the [0, 200] kA thunder and lightning flow is divided into 200 intervals according to $1 \mathrm{kA}$ as a step length. The lighting range and lighting stroke trip rate are calculated according to interval. The thunder lighting flow is not greatly different in each interval. The error of selecting the same lighting range is relatively small.

It is assumed that thunder and lightning can develop vertically downward approximately in a large number of studies. As overhead lines are higher than the ground, they have an attractive effect on thunder and lightning precursor. If the electric field intensity thereof with the line reaches the critical breakdown field strength of air, namely the distance between them reaches a certain fixed value, the line will suffer lightning stroke, the fixed value is defined as lightning stroke distance in the development of thunder and lightning precursor head to the ground. The model recommended by IEEE is used as shown in figure 1. The calculation formulas of conductor and ground lightning stroke distance are respectively shown as follows:

$$
\begin{aligned}
& r_{\mathrm{s}}=10^{0.65} \\
& r_{g}=0.9 r_{\mathrm{s}}
\end{aligned}
$$

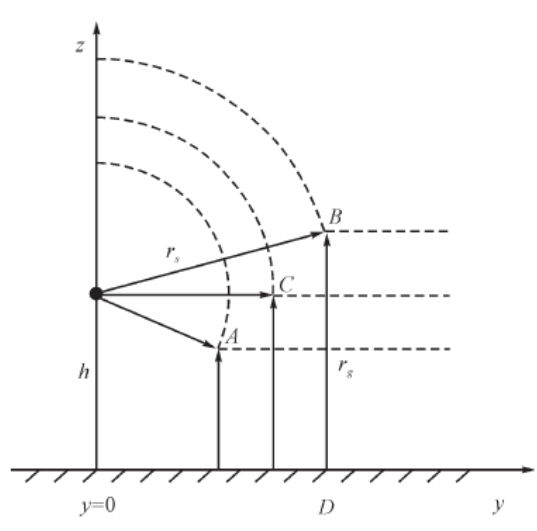

Fig. 1 Electrical geometric model 
When the lightning stroke line is close to the ground, the space electric field and magnetic field generated by the discharge channel will induce overvoltage on the conductor. If the induced overvoltage exceeds the withstand voltage of the line insulation, insulator flashover will be caused. Chinese regulations show that lighting strokes the ground when the distance between lightning stroke point and the line is $S>65 \mathrm{~m}$, and the induced overvoltage on the wire is shown as follows:

$$
\mathrm{U}_{\mathrm{i}}=\frac{25 \mathrm{Ih}}{S}
$$

The trip rate of inductive thunder $F_{I k}$ is calculated according to the following formula:

$$
F_{l k}=0.2 \gamma T_{d} \sum_{1}^{200}\left(\mathrm{y}_{\max , k}-D_{k}\right) \mathrm{P}_{k} \eta
$$

\section{Windage Yaw Flashover}

wind age yaw flashover is caused by wind deflection angle higher than the designed allowable value under wind load, thereby leading to insufficient electrical clearance among the charged parts (wires, wire clips, pressure equalizing rings, etc.) as well as between the charged part and the ground component or adjacent object, and flashover trip of the line is produced as a result. The charged body may still be in the state of wind deflection during recourse due to the continuity of wind load. The first-time flashover discharge increases free conductive ions in the air gap and reduces the insulation strength. The operating overvoltage generated by recourse may lead air gap flashover or breakdown again.

Influence of rainfall and lightning: The waterline formed by water vapor and rain in the air during typhoon reduces the flashover voltage of air gap, which is conducive to the occurrence of wind age yaw flashover. However, the influence of flashover voltage reduction under rainy condition is not considered in details during line design, thereby leading to low gap design. Typhoon is often accompanied by thunder and lightning, and the wind speed of thunder and lightning overvoltage gap of $15 \mathrm{~m} / \mathrm{s}$ is considered during tower design. The gust wind speed corresponding to actual working condition may exceed the value, so it is difficult for the line to meet the requirements of thunder and lightning overvoltage, and discharge is caused.

Phase-to-phase discharge occurs easily due to asynchronous swing of all phase conductors under continuous strong wind, micro-terrain, and conductor arc deviation during construction, the actual pitch exceeding the design value (the sag is related to the pitch), etc.

The ground wire has light dead weight and fast response speed. When the instantaneous wind exceeds the designed wind speed, and the conductors with large span swing upwards, the ground wire falls down from the maximum wind deflection angle, thereby resulting in distance reduction discharge between the ground wires. The arc error of the ground wire may lead to insufficient distance between the ground wires during construction pay-off. The ground wire distance is smaller than the safe gap, and discharge is caused when the wind deflection angle of the wire is relatively large.

\section{Insulator Wet Flashover}

When fouling insulators are wet, the soluble material contained in the fouling layer gradually dissolves in water, which becomes electrolyte. A thin conductive liquid film is formed on the surface of the insulator. The conductivity of the film depends on the chemical composition of the contaminant and the humidity. The surface resistance of the fouling layer drops by several orders of magnitude during wet and saturated condition. The leakage current of the insulator increases correspondingly. The heating is the most severe due to smaller diameter and maximum current density near the iron aangle. If the contaminant is serious or insulator creep age distance is small, the flash discharge current flowing through the drying belt is relatively large, the discharge channel is in the form of yellow red weave, it is coarser, the temperature is increased to thermal dissociation in the channel, which becomes arc discharge with falling volt-ampere characteristic, and the field intensity required 
for the channel is decreased. The voltage shared to the flashover discharge channel is sufficient to maintain long local electric arc without extinguishing. Finally, it is developed into discharge along the surface of the whole insulator.

The influence of rainfall on post insulator fouling rain flashover voltage also conforms to the power function relation as follows:

$$
\mathrm{k}_{1}=\mathrm{A}_{\mathrm{p}} \delta_{\text {water }}^{-\mathrm{a}}
$$

Wherein, $\delta_{\text {water }}$ refers to rainfall, $\mathrm{mm} / \mathrm{min} ; \mathrm{A}_{\mathrm{p}}$ refers to coefficient related to rainfall, a refers to the characteristic index of influence of characteristic rainfall on fouling rain flashover voltage; $\mathrm{k}_{1}$ refers to rainfall correction factor, namely the ratio between fouling rain flashover voltage of pillar insulator under different rainfall conditions and voltage with rainfall of $2 \mathrm{~mm} / \mathrm{min}$.

\section{Mechanical Failure}

\section{Tower Damage}

The main reasons of tower collapse and cross arm breaking caused by typhoon are design, topography, construction and other problems.

(1) The instantaneous wind speed exceeds the design value of line wind resistance. The new and old lines in operation are designed according to different rules. The requirements on re-occurrence period and tower wind load are also different. The typhoon above grade 12 made frequent landfall in coastal areas in recent years, and the central wind speed has greatly exceeded the maximum designed wind speed of the original lines, and it is the main reason for the tower collapse and crossarm breaking on the line. The wind speed is not uniform along the direction of tower height, line direction and vertical line direction. It is easy to cause uneven force of local and two-side conductors of the iron tower, and the conductors are twisted.

(2) Uneven span and large height difference; when there is a large height difference in one span (non-isolated pitch) or a large gap between the front and rear spans, the conductor and ground wire are affected by temperature change or wind pressure, relatively large unbalanced tension is generated, which is conveyed to the tower through hanging strings. The longitudinal unbalanced tension under typhoon weather is increased correspondingly; the actual comprehensive load on the tower is more than design level, thereby leading to damage on the tower.

(3) Micro topography; the sudden increase effect of wind speed should be obvious aiming at micro-topographic areas such as mountainous area wind tunnels, mountain passes, windward slopes, sea-facing canyons, etc., thereby leading to long period and large wire-swing and fatigue damage. Relatively large unbalanced tension leads to tower damage.

(4) Equipment quality and construction problems: power distribution network power pole producers have different quality levels. There are many quality problems, such as insufficient reinforcement of electric poles, surface cracks and weathering, etc. They are easy to break under the action of violent typhoon. In addition, there is problem of insufficient construction, such as insufficient depth of foundation embedding, improper protective measures, fastener loosening and fall-off, etc. Pole falling and breaking accidents are manifested as chain reaction caused by toppling of straight pole towers mainly, and its main reason lies in frequent damage or missing of stay wires.

\section{Foundation Damage}

Foundation damage is usually caused by secondary disasters due to typhoon, such as landslides caused by heavy rain with typhoon, which may damage the tower foundation in these areas. In 2008, violent typhoon 'Hagupit' caused protective layer peel- off of some iron tower foundations, bottom surface tunneling and other accidents in $110 \mathrm{kV}$ flat-gate A-B line. 


\section{Conclusion}

Because the insulation level of distribution line is generally low, lightning stroke failures of distribution line mainly include induced overvoltage. Distribution line not only has low self-insulation level, but also encounters problems of many lines and complicated line topology. The possibility of insulator flashover is increased due to industrial and marine salt pollution in coastal areas due to industrial development and shorter distance from the sea.

When typhoon comes, strong wind is the direct cause of wind age yaw flashover. Small design margin is its internal cause. Proper improvement of design wind speed can effectively reduce the occurrence of wind age yaw flashover.

Mechanical failure is caused by typhoon mainly because wind force of the typhoon exceeds the design standard of line wind load, and the influence on micro topography and microclimate is relatively high. Meanwhile, there are influences due to equipment quality, insufficient wind-resistant stay wires, etc. Mechanical failure is the most extensive and difficult to recover in a short time in the influence caused by typhoon. Therefore, prevention of mechanical failures, such as tower falling, wire breaking, etc., becomes the most important task to prevent typhoon damage to distribution lines.

\section{References}

[1] LIANG Xiaoyun, GUO Yanjun. Global Significant Climate Events in 2007 [J]. Meteorological Monthly, 2008, 34(4):113-117.

[2] PANG Zhun, LI Bangfeng, YU Yue, et al.Study on Operating Modes of Hainan Power Grid during Typhoon Periods [J].Power System Technology,2007,31(7):46-50.

[3] SONG Jianwu, WANG Zhimin, CHEN Shilou. Enlightenment about Waterlogging Loss not seen once in a Hundred Years on Distribution Network in Fuzhou City [J]. Power Demand Side Management, 2006, 8(3):52-53.

[4] JIANG Liping, Xia Guancong, You Hong, et al. Analysis on the Abnormal Intention and Track of Typhoon "Chanchu” [J]. Journal of Oceanography in Taiwan Strait, 2008, 27(1):124-128.

[5] ZHANG Feng, WU Qiuhan, LI Jihong. Hazards of Typhoon Rananim to Zhejiang Power Grid And Its Preventive Measures [J]. Electric Power, 2005, 38(5):39-42.

[6] ZHANG Yong. Status quo of Wind Hazard Prevention for Transmission Lines and Countermeasures [J]. East China Electric Power, 2006, 34(3):28-31. 